\title{
RÉPARTITION DES ÉCREVISSES À PATTES BLANCHES, AUSTROPOTAMOBIUS PALLIPES (LEREBOULLET, 1858) DANS TROIS RUISSEAUX DE CORRĖZE ; OBSERVATION PARTICULIĖRE DES JUVÉNILES.
}

\author{
Y. REYJOL (1), C. ROQUEPLO (2)
}

(1) Centre d'Etude des Systèmes Aquatiques Continentaux (CESAC), CNRS 5576, Université Paul Sabatier, 118 route de Narbonne, 31062 TOULOUSE Cedex 4, France. E-mail : yorick.reyjol@ifrance.com

(2) Cemagref, Unité Ressources Aquatiques Continentales, 50 avenue de Verdun, 33612 CESTAS Cedex, France. E-mail : charles.roqueplo@bordeaux.cemagref.fr

\section{RÉSUMÉ}

L'habitat préférentiel des écrevisses à pattes blanches Austropotamobius pallipes (Lereboullet, 1858) a été analysé dans trois cours d'eau de Corrèze, grâce à la combinaison de trois méthodes de capture (pêche au filet de type Surber, pêche électrique et pêche à la main de nuit).

Parmi ces méthodes, la plus efficace pour la capture des juvéniles s'est avérée être le filet de type Surber, qui a permis la capture de 536 individus. Suite à cette étude, il apparaît que les écrevisses adultes recherchent de préférence les berges constituées de racines ou avec des cavités, les blocs, les galets, la végétation aquatique et les souches pour s'abriter durant le jour, tandis qu'elles fréquentent préférentiellement les graviers, les galets et les blocs pendant leurs déplacements nocturnes. Les juvéniles s'abritent de préférence dans les galets, les blocs, les végétaux aquatiques et les berges composées d'amas de radicelles. Dans certains cas, la réduction, voire la disparition d'un habitat particulièrement favorable aux juvéniles peut être compensée par une colonisation plus importante d'autres habitats habituellement délaissés. habitat.

Mots-clés : Astacidae, Austropotamobius pallipes, échantillonnage, juvéniles, 


\title{
PREFERENTIAL HABITAT ANALYSIS OF WHITE-CLAWED CRAYFISH, NOTABLY JUVENILES, IN THREE BROOKS OF CORRĖZE - FRANCE.
}

\begin{abstract}
The use of three capture methods (Surber sampling, electric fishing and night-hand capture) allowed the analysis of differential habitat usage by the white-clawed crayfish Austropotamobius pallipes (Lereboullet, 1858) in three brooks of Corrèze - France.

Among these methods, the best one for studying juveniles habitat was Surber sampling, which allowed the capture of 536 individuals. This study showed that, during daytime, most of the adults occurred in banks with crevices or roots, areas of pebbles or boulders, those with a high macrophytic covering and also in logs, while they were preferentially found in areas of gravels, pebbles and boulders during their nocturnal movements. The young of the year were caught especially among pebbles, boulders, macrophytes and river banks with rootlets. In some cases, the reduction or disappearance of one habitat which is particularly favourable to the young of the year might be compensated by a more active colonization of some habitats which normally shelter only a few crayfish.
\end{abstract} habitat.

Key-words: Astacidae, Austropotamobius pallipes, sampling, young-of-the-year,

\section{INTRODUCTION}

Les observations réalisées par le Conseil Supérieur de la Pêche lors des enquêtes sur la répartition des populations d'écrevisses en France (ARRIGNON, 1990) ont montré que Austropotamobius pallipes (Lereboullet, 1858) peut coloniser des milieux ayant des caractéristiques différentes (cours d'eau, plan d'eau avec une forte ou une faible minéralisation de l'eau, etc.). Si cette espèce est effectivement présente sur une grande partie de notre territoire (72 départements en 1990), le nombre de ses populations ainsi que leur densité sont en constante régression. Ce phénomène s'observe également à l'échelle européenne (HOGGER, 1988). Cette régression peut avoir plusieurs causes : la surexploitation halieutique, l'introduction d'espèces allochtones agressives et porteuses saines de germes pathogènes, la dégradation de certains paramètres de l'environnement aquatique et la modification des habitats disponibles.

L'habitat préférentiel des écrevisses de plus de 1 an a fait l'objet de nombreuses analyses (ARRIGNON et ROCHE, 1981 ; DAGUERRE DE HUREAUX et ROQUEPLO, 1981 ; ROQUEPLO, 1983, 1997, 1999 ; LACHAT et LAURENT, 1987 ; FOSTER, 1995 ; SMITH et al., 1995 ; NEVEU, 1996 ; DAVENNE et FIRMIGNAC, 1997), mais les études intégrant l'habitat des juvéniles restent rares (NEVEU, 2000). Généralement, les résultats obtenus sont basés sur la capture d'un nombre restreint d'individus, du fait de méthodes non adaptées à l'échantillonnage de ces jeunes stades (BROWN et BOWLER, 1976 ; BREWIS, 1978 ; NEVEU, 1996), qui restent enfouis dans le substrat.

Des travaux menés depuis plusieurs années dans le département de la Corrèze (ROQUEPLO, 1999) ont montré que des populations d'écrevisses à pattes blanches sont localement bien implantées dans plusieurs cours d'eau. L'analyse des relations entre cette espèce et son environnement proche a montré que la diversité des biotopes aquatiques 
ainsi que la faible pression anthropique sur le proche bassin versant sont deux facteurs essentiels favorisant son implantation.

Notre étude analyse la répartition des écrevisses dans trois ruisseaux de Corrèze. Une bonne connaissance des habitats préférentiellement recherchés par les écrevisses du stade juvénile jusqu'au stade adulte est indispensable pour comprendre la colonisation d'un cours d'eau par cette espèce et proposer des stratégies de gestion favorisant l'implantation de ses populations d'écrevisses.

\section{MÉTHODES D'ÉTUDE}

\section{Localisation et description des sites}

Les trois ruisseaux étudiés (le Vent Haut, le Pont Aubert et le Lachaud) sont localisés à $15 \mathrm{~km}$ au sud de Neuvic d'Ussel, en Corrèze. Cette région de moyenne montagne est située au Nord-Est du département, à environ 600 mètres d'altitude (Figure 1).

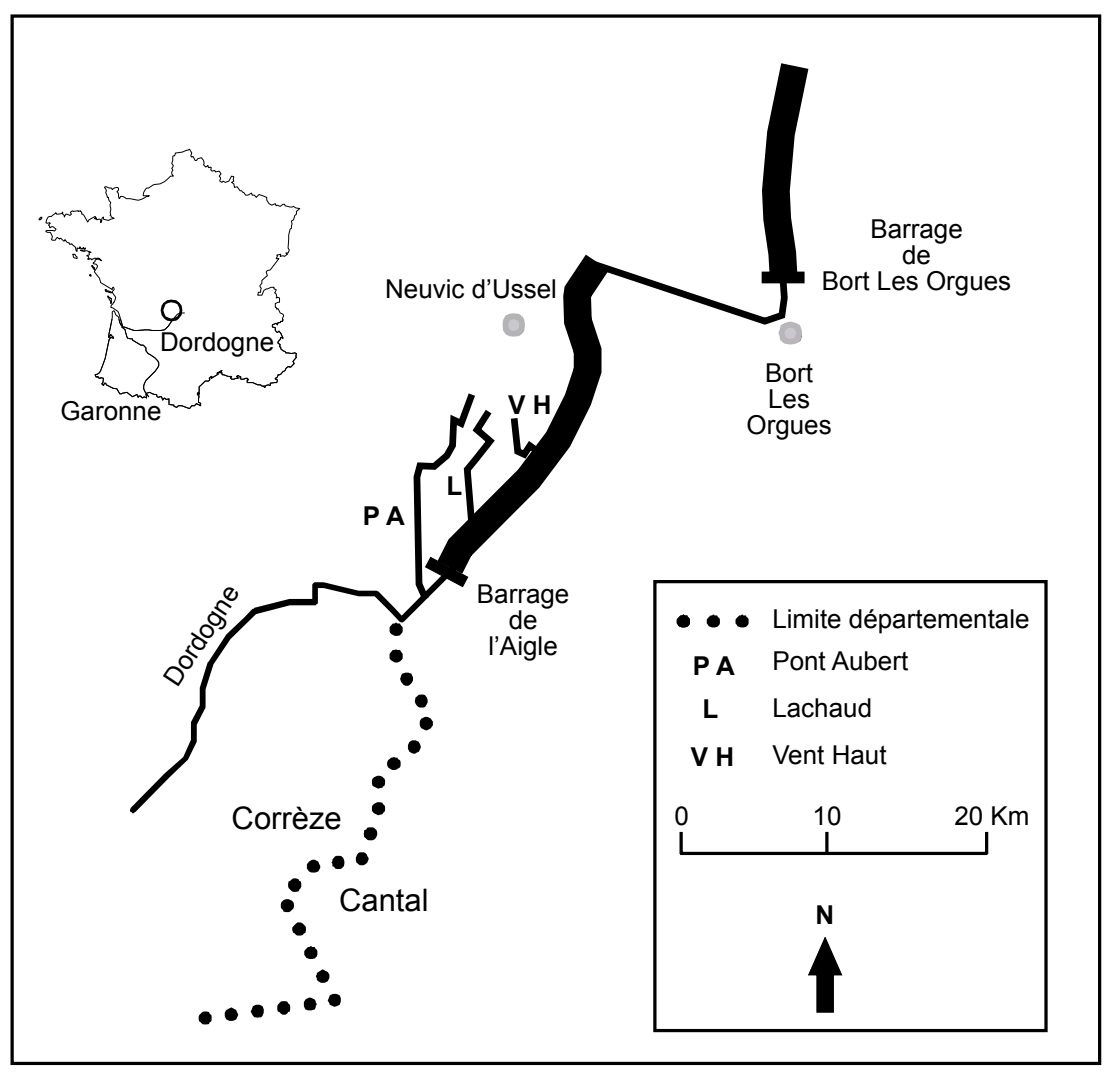

\section{Figure 1}

Localisation des ruisseaux étudiés dans le département de la Corrèze.

Figure 1

Localisation of the studied brooks in the Department of Corrèze. 
- Le Vent Haut, ruisseau de 0,8 à $3 \mathrm{~m}$ de large, n'abrite comme faune aquatique que des larves d'insectes et des écrevisses. Son cours fortement encaissé dans une hêtraie suit une pente de $14 \%$ en moyenne. Après un parcours d'un peu plus de $1 \mathrm{~km}$, se terminant par plusieurs cascades, il rejoint la Triouzoune, affluent de la Dordogne, au niveau de la retenue de l'Aigle.

- Le Pont Aubert, de 2 à $4 \mathrm{~m}$ de largeur et $10 \mathrm{~km}$ de long pour une pente moyenne de $3 \%$, coule dans une petite vallée caractérisée par l'implantation de plusieurs villages. II a fait l'objet d'un nettoyage de ses berges et de son lit de 1995 à 1997. Ces travaux, tout en permettant un meilleur écoulement de l'eau, ont fortement diminué la diversité des abris potentiellement utilisables par les poissons et les écrevisses. L'activité agricole principale étant l'élevage de bovidés et la production laitière, le Pont Aubert subit une pollution légère par un apport chronique de matière organique.

- Le Lachaud, avec une largeur de 1 à 2,8 $\mathrm{m}$ pour 4,5 $\mathrm{km}$ de long et une pente de $3,7 \%$ en moyenne, serpente dans des fonds de vallons boisés, où l'activité humaine ne se remarque que par des prairies destinées à l'élevage des bovidés.

Les stations d'étude sur ces trois cours d'eau ont été choisies en fonction de la diversité des paramètres morphologiques, afin de pouvoir échantillonner tous les habitats préalablement recensés.

Sur le Vent Haut, 3 stations d'une surface totale de $146 \mathrm{~m}^{2}$ ont pu être identifiées. La profondeur y est de 5 à $15 \mathrm{~cm}$ dans les zones courantes et 40 à $50 \mathrm{~cm}$ dans les deux zones profondes. Le ruisseau, constamment ombragé, coule sur un lit de galets et de sable, avec de nombreux blocs près des berges.

Sur le Pont Aubert, 2 stations de $13 \mathrm{~m}$ et de $60 \mathrm{~m}$ de long ont permis d'échantillonner $156 \mathrm{~m}^{2}$. La profondeur est comprise entre 5 et $10 \mathrm{~cm}$ pour les zones courantes et de $40 \mathrm{~cm}$ dans une zone profonde de moins de $1 \mathrm{~m}^{2}$ près d'une berge. Ces stations sont situées dans la partie amont du cours d'eau et bénéficient de berges boisées. Par ailleurs, le nettoyage des berges a permis un bon développement de la végétation aquatique. Le lit de ce cours d'eau est dominé par le sable, mais compte également de nombreux graviers, galets et blocs.

Sur le Lachaud, 2 stations d'échantillonnage avec des zones de courant de 5 à $10 \mathrm{~cm}$ de hauteur d'eau et une zone profonde de $50 \mathrm{~cm}$ ont permis d'échantillonner $117 \mathrm{~m}^{2}$. La rive gauche de ces stations est occupée par des bois, tandis que la rive droite est dominée par des pâturages. Le lit du ruisseau est largement dominé par des galets et du sable.

\section{Echantillonnage des écrevisses}

La capture des jeunes individus nés dans l'année s'est déroulée essentiellement du 16 juin au 30 juillet 1998. Dans ces ruisseaux, l'éclosion des écrevisses à pattes blanches se situe, en général, entre la fin du mois de mai et le début du mois de juin (ROQUEPLO, 1999) et la taille des juvéniles pendant la période d'échantillonnage y est comprise entre 8 et $16 \mathrm{~mm}$.

Les moyens traditionnels de capture des écrevisses adultes (balances, nasses, pêche à la main, pêche électrique) sont mal adaptés à l'échantillonnage des juvéniles (ROQUEPLO, 1992). Du fait de leur petite taille et de leur tendance à se cacher dans le substrat, les écrevisses juvéniles montrent des similitudes avec les macro invertébrés benthiques. Plusieurs outils existent pour l'échantillonnage de ce type d'organismes : troubleaux, bennes, aspirateurs, filets de type Surber, etc. (MACAN, 1958). Parmi ceux-ci, nous avons choisi d'utiliser un petit filet benthique, avec une maille de 0,5 mm, inspiré du filet de type Surber, pour l'échantillonnage des juvéniles d'écrevisses. 
L'unité d'échantillonnage avec le filet benthique, matérialisée par un cadre en métal, était de $0,1 \mathrm{~m}^{2}$ lorsque la surface des habitats était suffisante. Sinon, elle était réduite à $0,02 \mathrm{~m}^{2}$, ce qui nous a permis de multiplier les prélèvements dans les habitats représentés par des surfaces de quelques décimètres carrés seulement.

Pour capturer un plus grand nombre d'individus de plus de $6 \mathrm{~cm}$, les inventaires par filet benthique ont été complétés par :

- La pêche à l'électricité (200 volts pour 0,5 à 0,8 ampère), qui permet d'attirer les écrevisses vers l'anode (WESTMAN et al., 1978) et de les faire sortir de leurs abris. II est cependant indispensable de maintenir le champ électrique pendant une durée supérieure à celle généralement utilisée pour la capture des poissons, soit pendant 20 à 30 secondes, pour avoir un effet attractif correct sur les écrevisses.

- La pêche à la main de nuit, qui a été pratiquée d'aval en amont, sur chaque station, de 21 h 30 à minuit, ce qui correspond à la période maximale d'activité de cette espèce dans les cours d'eau (FENOUIL et CHAIX, 1985).

L'utilisation de ces trois méthodes de capture permet d'échantillonner :

- Les habitats utilisés dans la journée par les écrevisses, pour leur protection (abris vis à vis de la lumière et contre les prédateurs).

- Les zones d'activité utilisées la nuit par les écrevisses, pour l'alimentation.

Les écrevisses capturées ont été mesurées de la pointe du rostre à l'extrémité du telson, au demi-millimètre près, en les disposant sur une règle spéciale. Les écrevisses ont ensuite été relâchées à l'endroit de leur capture, en évitant qu'elles soient emportées par le courant.

En tenant compte des observations déjà réalisées sur ces cours d'eau, nous avons regroupé les tailles des écrevisses en quatre classes :

- Juvéniles, pour les individus venant d'éclore et ayant une longueur totale comprise entre 8 et $16 \mathrm{~mm}$ au début de l'été.

- Jeunes, pour les écrevisses nées l'année précédente et ayant une taille comprise entre 17 et $30 \mathrm{~mm}$.

- Sub-adultes, pour toutes les écrevisses entre 31 et $60 \mathrm{~mm}$; la maturation sexuelle sur ces ruisseaux étant atteinte entre 55 et $60 \mathrm{~mm}$, ce qui est conforme avec des observations réalisées pour d'autres populations (ROQUEPLO, 1983 ; FENOUIL, 1987 ; NEVEU, 1996).

- Adultes, pour toutes les écrevisses d'une taille supérieure à $60 \mathrm{~mm}$.

\section{Stratification de l'habitat et plan d'échantillonnage}

Afin de comparer les différents habitats occupés par les juvéniles, nous avons pratiqué un échantillonnage de type stratifié (FRONTIER, 1983). Cette méthode consiste à subdiviser une population hétérogène (les stations) en sous-populations ou strates plus homogènes (les différents habitats) et à y effectuer un échantillonnage au hasard, afin de ne pas oublier certains habitats minoritaires. Notre plan d'échantillonnage a porté uniquement sur la nature de l'habitat, et n'a pas tenu compte des paramètres comme la vitesse d'écoulement et la hauteur d'eau. En effet, ceci aurait abouti à la stratification des stations en de nombreuses zones de petites surfaces et aurait posé des problèmes lors de l'échantillonnage. 
Chaque station a fait l'objet d'un relevé topographique, qui a permis de mesurer les dimensions des différents habitats identifiés.

Nous avons ainsi identifié et étudié 8 habitats :

- Sable : diamètre $<1 \mathrm{~mm}$.

- Graviers : de $1 \mathrm{~mm}$ à $2 \mathrm{~cm}$.

- Galets : de 2,1 cm à $10 \mathrm{~cm}$.

- Blocs : > $10 \mathrm{~cm}$.

- Berges.

- Végétaux aquatiques : Genres Myriophyllum et Fontinalis.

- Souches.

- Débris végétaux (essentiellement des feuilles et des morceaux de branches).

\section{Rythme d'échantillonnage}

Nous avons choisi de réaliser 10 prélèvements par type d'habitat dans chaque ruisseau, afin de permettre un traitement statistique de toutes les données, sous SYSTAT 6.0.2. En cas de faible représentativité d'un habitat particulier, le nombre d'échantillons correspondant a pu être occasionnellement réduit. Compte tenu des perturbations provoquées sur le fond des ruisseaux par nos campagnes de prélèvements, nous avons respecté un intervalle de trois jours entre deux échantillonnages successifs. Une centaine d'échantillons a pu être réalisée par type d'habitat dans chaque cours d'eau.

\section{RÉSULTATS}

\section{Stations d'étude}

Le substrat granitique de cette région conditionne la qualité physico-chimique des eaux de tous les ruisseaux, qui ont pour caractéristique commune une faible minéralisation se traduisant par des teneurs en calcium dissous particulièrement faibles, comprises entre 1,2 et $8,5 \mathrm{mg} / \mathrm{L}$ (Tableau I).

\section{Tableau I}

Valeurs des principaux paramètres physico-chimiques analysés sur les trois ruisseaux. Résultats des dosages en $\mathrm{mg} / \mathrm{L}$, excepté pour la conductivité $(\mu \mathrm{S} / \mathrm{cm})$ et le $\mathrm{pH}$.

\section{Table I}

Values of the main physico-chemical parameters in the three brooks. The measuring out results are in $\mathrm{mg} / \mathrm{L}$, except for conductivity $(\mu \mathrm{S} / \mathrm{cm})$ and $\mathrm{pH}$.

\begin{tabular}{|c|c|c|c|}
\hline & Moyenne & Minimum & Maximum \\
\hline Conductivité & 40,00 & 6,00 & 66,00 \\
\hline pH & 7,33 & 6,50 & 7,60 \\
\hline Oxygène & 7,57 & 6,50 & 8,20 \\
\hline Oxydabilité & 10,67 & 8,10 & 12,20 \\
\hline Calcium & 7,67 & 1,20 & 8,50 \\
\hline Magnésium & 1,17 & 0,90 & 2,30 \\
\hline Nitrates $\left.\left(\mathrm{NO}_{3}\right)^{-}\right)$ & 3,67 & 0 & 4,50 \\
\hline Nitrites $\left(\mathrm{NO}_{2}\right)^{-}$ & 0,03 & 0 & 0,05 \\
\hline Ammonium $\left(\mathrm{NH}_{4}^{+}\right)$ & 0,06 & 0 & 0,10 \\
\hline Orthophosphates (en $\mathrm{PO}_{4}^{3-}$ ) & 0,02 & 0,01 & 0,03 \\
\hline Sulfates $\left(\mathrm{SO}_{4}{ }^{2-}\right)$ & 0 & 0 & 0 \\
\hline Chlorure (Cl) & 7,67 & 7,20 & 8,10 \\
\hline
\end{tabular}


La température de ces trois cours d'eau est relativement fraîche. En hiver la température moyenne de l'eau est proche de $5^{\circ} \mathrm{C}$, avec toutefois des amplitudes thermiques quotidiennes pouvant atteindre $4^{\circ} \mathrm{C}$. Elle passe en dessous de $10^{\circ} \mathrm{C}$ à partir de la mi-octobre, jusqu'à la mi-avril dans le Vent-Haut et un mois plus tôt dans les deux autres ruisseaux. En été, la température se stabilise entre 14 et $16^{\circ} \mathrm{C}$ dans le Vent-Haut et peut atteindre 18 à $20^{\circ} \mathrm{C}$ dans le Pont Aubert et le Lachaud.

\section{Répartition des habitats}

La représentation des différents habitats n'est pas identique sur chacun des trois ruisseaux (Tableau II). Ainsi, les végétaux aquatiques sont absents du Vent Haut, du fait du fort ombrage, et les zones de graviers qui sont communes dans le Pont Aubert sont inexistantes dans le Lachaud.

\section{Tableau II}

Surface des habitats $\left(\mathrm{m}^{2}\right)$ et longueur de berges $(\mathrm{m})$ pouvant abriter des écrevisses, sur les stations des trois cours d'eau.

\section{Table II}

Area for each habitat $\left(\mathrm{m}^{2}\right)$ and length of river banks $(\mathrm{m})$ in the stations of the three brooks which could be occupied by crayfish.

\begin{tabular}{|c|c|c|c|c|c|c|c|c|c|}
\cline { 2 - 9 } \multicolumn{1}{c|}{} & Sable & Graviers & Galets & Blocs & Végétation & Berges & Souches & Débris & $\begin{array}{c}\text { Surface } \\
\text { totale }\end{array}$ \\
\hline Pont Aubert & 89 & 13,5 & 34,5 & 18 & 18 & 50,5 & 0,8 & 9,5 & 183,3 \\
\hline Lachaud & 28,5 & 0 & 79 & 5,5 & 9 & 98,5 & 0,5 & 1,5 & 124 \\
\hline Vent Haut & 19 & 3 & 33,5 & 9,5 & 0 & 6 & 5 & 1,5 & 71,5 \\
\hline Total & $\mathbf{1 3 6 , 5}$ & $\mathbf{1 6 , 5}$ & $\mathbf{1 4 7 , 0}$ & $\mathbf{3 3 , 0}$ & $\mathbf{2 7 , 0}$ & $\mathbf{1 5 5 , 0}$ & $\mathbf{6 , 3}$ & $\mathbf{1 2 , 5}$ & $\mathbf{3 7 8 , 8}$ \\
\hline
\end{tabular}

\section{Capture des écrevisses}

Nous avons pu échantillonner au total 1157 écrevisses, dont 538 juvéniles, en utilisant les trois techniques de pêche. La comparaison globale des résultats d'après la taille des écrevisses (test $\mathrm{t}$ ) met en évidence des différences hautement significatives $(p<0,01)$ entre les trois méthodes de pêche (Figure 2).

Le filet benthique permet surtout la capture d'écrevisses juvéniles, mais permet également la capture d'écrevisses de plus grande taille, comme les sub-adultes. Globalement, plus la taille des écrevisses augmente et plus la fréquence des captures dans le filet benthique diminue.

La pêche à l'électricité présente une efficacité maximale pour les écrevisses ayant une taille supérieure à $3 \mathrm{~cm}$. Près de la moitié des écrevisses capturées par cette méthode sont des sub-adultes.

La pêche à la main de nuit présente une efficacité maximale pour les adultes (42\% des captures), mais ne permet pas d'échantillonner correctement les juvéniles. 


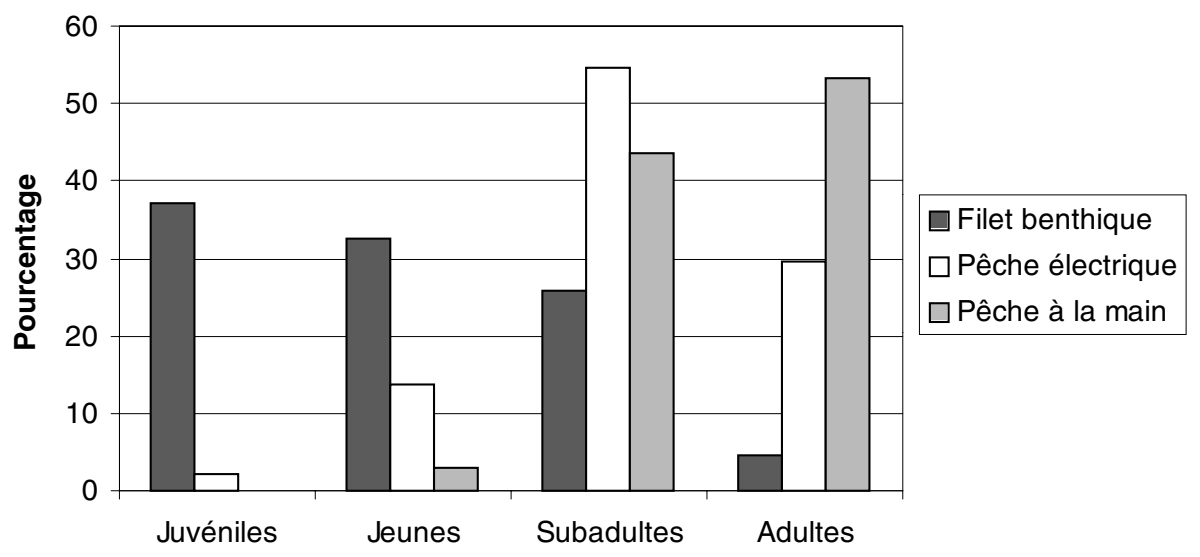

\section{Figure 2 \\ Répartition des classes de taille d'écrevisse en fonction de la méthode de capture.}

\section{Figure 2}

Distribution of crayfish lengths in relation to the catching method.

\section{Répartition des écrevisses dans les différents habitats}

Globalement, les écrevisses sont présentes dans tous les habitats identifiés sur les trois cours d'eau, mais une grande disparité existe entre les résultats, suivant la taille des écrevisses et la méthode de capture.

\section{Echantillonnage par filet benthique}

Vingt-huit campagnes de prélèvement au filet benthique ont permis de réaliser 1630 échantillons dans les différents habitats. 886 écrevisses dont 536 juvéniles ont été capturées par cette méthode, soit $60,5 \%$ du nombre total d'individus (Figure 3). Lors des prélèvements, une mortalité liée aux manipulations n'a pu être évitée pour les juvéniles, compte tenu de leur faible taille et de leur fragilité. Cependant, avec $4,6 \%$ en moyenne, elle reste faible.

En fonction de la taille des écrevisses capturées, les divers habitats échantillonnés par le filet benthique peuvent être séparés en :

- Des zones presque complètement délaissées par les écrevisses. Ce sont le sable, les graviers et les débris végétaux.

- Les souches, qui constituent une zone difficilement échantillonnable avec cet engin de capture et qui n'a permis d'y récolter qu'un très faible nombre d'écrevisses.

- Des habitats dominés par la présence de juvéniles. Ce sont les galets et les plantes aquatiques, avec plus de 140 individus dans chacun d'eux.

- Des habitats où l'on peut rencontrer des écrevisses de toutes les tailles, depuis les juvéniles jusqu'aux adultes. Ce sont les blocs et les berges. 


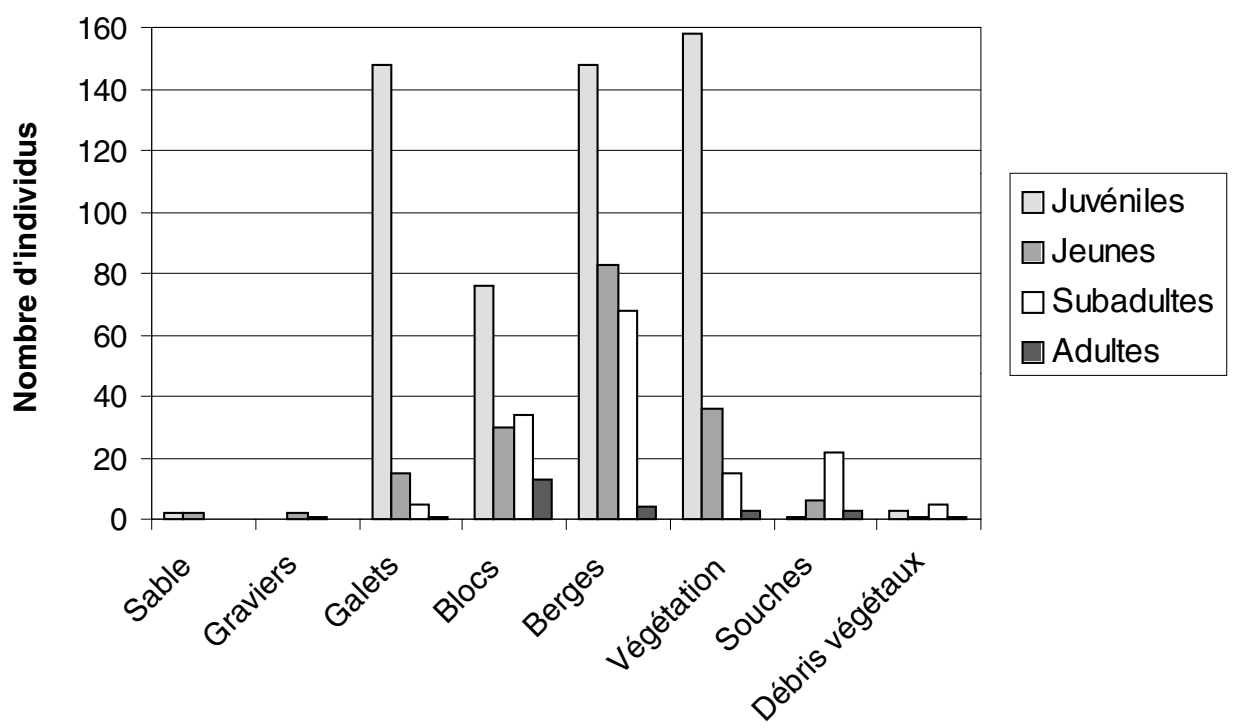

Figure 3

Répartition globale des écrevisses capturées au filet benthique dans les différents habitats.

\section{Figure 3}

Global distribution of crayfish caught with the benthic net in the different habitats.

\section{Echantillonnage par pêche électrique}

La pêche électrique pratiquée en cours de journée permet la capture des écrevisses dans leurs abris et donc l'analyse de la colonisation des habitats utilisés pour le repos et la protection. Plus de $80 \%$ des écrevisses échantillonnées par cette méthode (137 individus au total) ont été des adultes et des sub-adultes (Figure 4).

En fonction de la taille des écrevisses capturées, les divers habitats échantillonnés par pêche électrique peuvent être une nouvelle fois séparés : écrevisses.

- Le sable, le gravier et la végétation sont les habitats les plus pauvres en

- Les galets, les blocs, les souches et les débris végétaux abritent la plupart des sub-adultes.

- Les berges sont colonisées par un grand nombre d'adultes.

Echantillonnage par pêche à la main de nuit

Cette technique de capture permet d'échantillonner les diverses zones fréquentées par les écrevisses lors de leur activité nocturne, essentiellement pour la recherche de nourriture. La pêche à la main de nuit, en capturant les écrevisses en déplacement sur le fond du ruisseau, est une méthode qui ne permet pas de capturer les juvéniles de l'année. L'essentiel de nos observations a donc porté sur les sub-adultes et les adultes, ces derniers constituant $61 \%$ des captures (134 individus au total) (Figure 5).

Ainsi, les adultes et les sub-adultes se déplacent fréquemment dans les zones correspondant aux graviers, aux galets et aux blocs. 


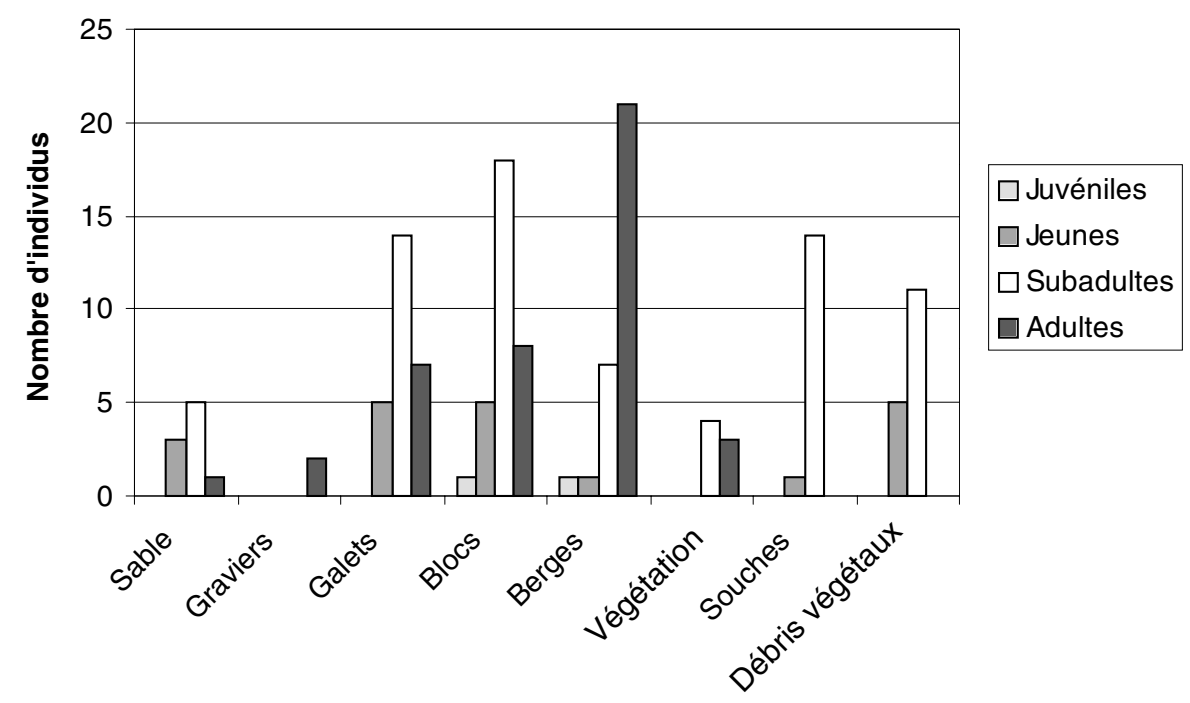

Figure 4

Répartition globale des écrevisses capturées par pêche électrique dans les différents habitats.

\section{Figure 4}

Global distribution of crayfish caught by electric fishing in the different habitats.

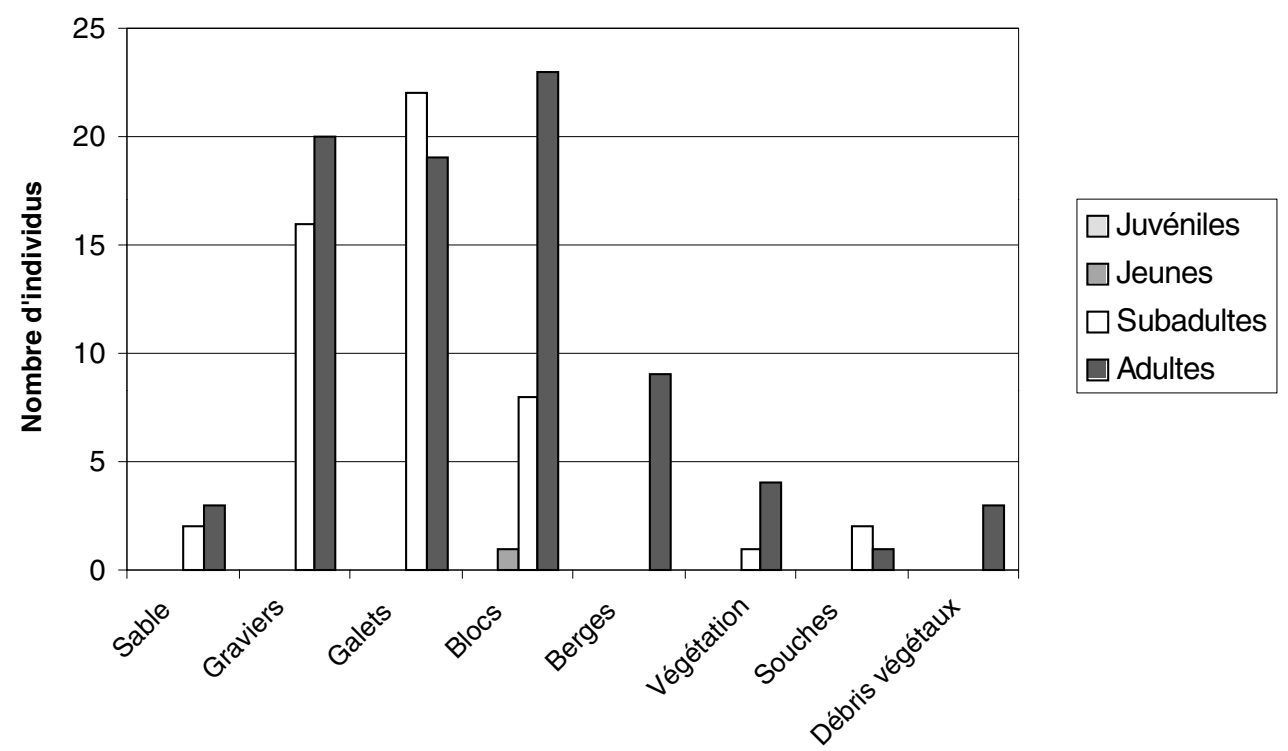

Figure 5

Répartition globale des écrevisses capturées de nuit à la main dans les différents habitats.

\section{Figure 5}

Global distribution of crayfish caught by night-hand capture in the different habitats. 


\section{Zones de refuge et zones de déplacement des écrevisses adultes}

La présence des écrevisses adultes dans les habitats utilisés pour leur protection en cours de journée peut être appréhendée par la pêche à l'électricité et par le filet benthique (Figure 6). Pour chaque habitat utilisé en tant que refuge, des densités de capture ont pu être calculées en tenant compte des surfaces échantillonnées. Pour le calcul des densités dans les berges, nous avons considéré la longueur de celles-ci multipliée par la largeur moyenne échantillonnée, de l'ordre de $1 \mathrm{~m}$.

L'activité des écrevisses en déplacement est évaluée par les captures nocturnes à la main.

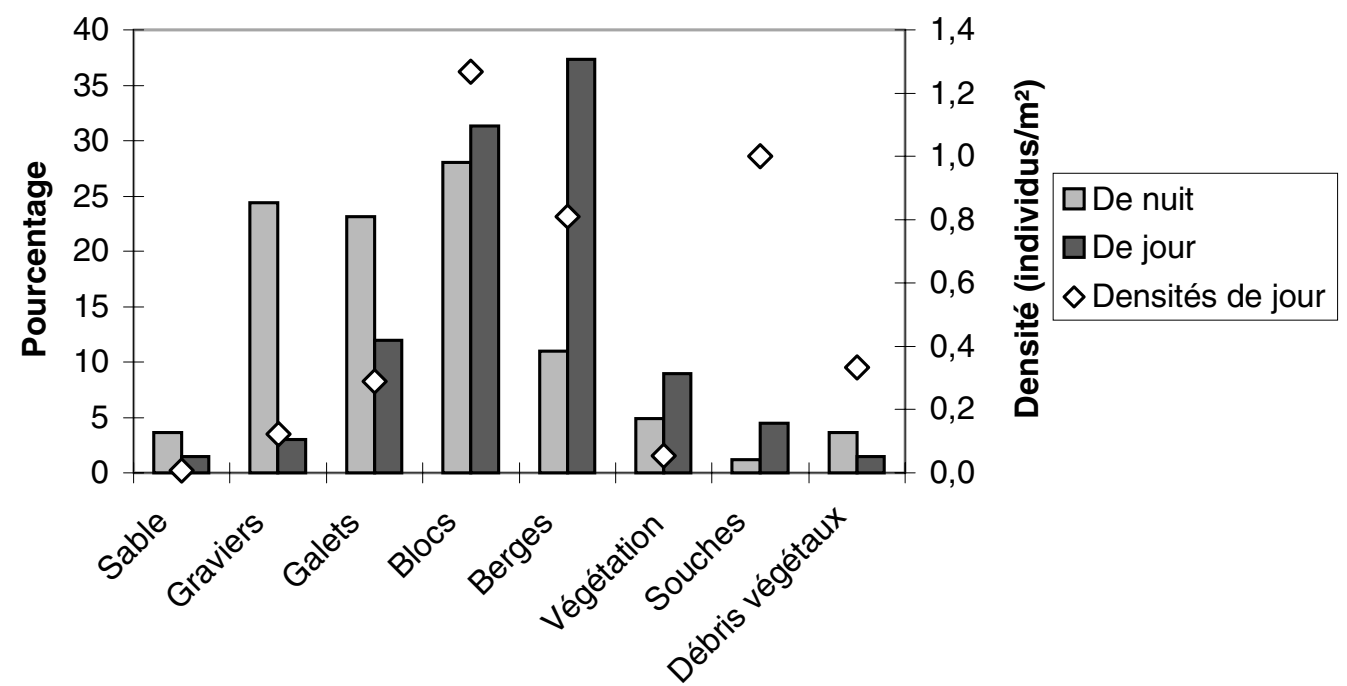

Figure 6

Utilisation des différents habitats par les écrevisses adultes. Résultats exprimés en pourcentage de présence dans les habitats, en fonction du comportement des écrevisses (jour ou nuit) et en densité de capture (individus $/ \mathrm{m}^{2}$ ) dans les habitats refuge par échantillonnage au filet Surber.

\section{Figure 6}

Use of the different habitats by adult crayfish. Results are in percentage of presence in the habitats, depending on the behaviour of crayfish (night or day) and in catch densities (individuals $/ \mathrm{m}^{2}$ ) in the habitats used as shelters for the Surber sampling.

La répartition des écrevisses présente une différence très hautement significative entre la fréquence des captures d'adultes dans les habitats fréquentés pour les déplacements nocturnes et ceux utilisés en tant que refuges $\left(\chi^{2}=29,01\right.$ pour $\left.\mathrm{ddl}=7\right)$.

Les habitats échantillonnés peuvent être regroupés en :

- Des habitats fréquentés par les écrevisses pendant leurs déplacements nocturnes : les graviers, les galets et les blocs.

- Des habitats recherchés par les adultes en tant qu'abris : les berges, les blocs, les galets, la végétation aquatique et les souches. végétaux.

- Des habitats peu fréquentés par les écrevisses adultes : le sable et les débris 


\section{Colonisation des berges par les écrevisses}

Les berges constituent un habitat complexe, qui, en fonction de la nature et de la dimension des éléments qui les composent, peut être scindé, dans les ruisseaux de Corrèze, en 6 unités homogènes (chacune d'elles ayant pu être appréhendée en mètre linéaire puis multipliée par une largeur d'échantillonnage de $1 \mathrm{~m}$ en moyenne afin d'en calculer la surface) :

- Les berges faites d'enchevêtrements de racines de diamètre supérieur à $1 \mathrm{~cm}$.

- Les berges tapissées de radicelles, généralement d'aulnes.

- Les berges verticales constituées d'argile ou d'autres dépôts sédimentaires avec une végétation herbacée abondante.

- Les berges avec des cavités plus ou moins profondes (> $10 \mathrm{~cm})$.

- Les berges avec des cavités garnies de galets non jointifs.

- Les berges constituées d'un empilement de galets ou de blocs non jointifs.

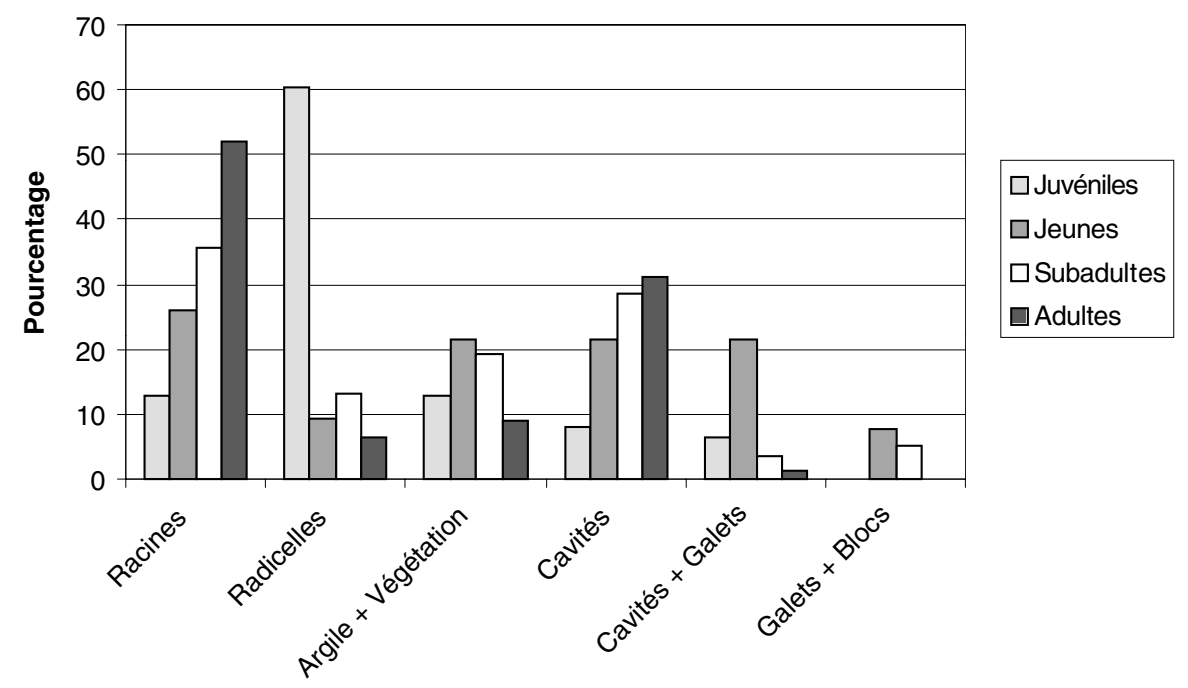

\section{Figure 7}

Colonisation des différents types de berge par les écrevisses de chaque stade.

\section{Figure 7}

\section{Colonization of the different types of bank by crayfish of each stage.}

La répartition des écrevisses dans les 6 types de berge échantillonnées présente des différences très hautement significatives $\left(\chi^{2}=274\right.$, pour $\left.\mathrm{ddl}=15\right)$

II est possible d'identifier :

- Des berges peu colonisées par les écrevisses : elles sont constituées de galets ou de blocs ainsi que de cavités contenant des amas de galets.

- Des berges abritant toutes les catégories d'écrevisses : ce sont les racines de plus de $1 \mathrm{~cm}$ de diamètre, les berges en argile recouverte de végétation ainsi que les cavités.

- Les amas de radicelles peuvent être considérés comme un type de berge à part, largement dominé par les juvéniles (60\% des cas). 


\section{Habitats préférentiels des juvéniles}

La répartition des juvéniles dans les différents habitats a pu être abordée essentiellement par les captures effectuées avec le filet benthique (Figure 3).

L'analyse des résultats d'échantillonnage montre qu'il y a bien une différence très hautement significative dans la fréquence des juvéniles entre les différents habitats $\left(\chi^{2}=343\right.$, pour $\left.\mathrm{ddl}=14\right)$.

Connaissant la surface des échantillons réalisés dans chaque habitat sur les différents ruisseaux, il est possible d'estimer la densité des juvéniles dans les captures et donc de comparer le taux de colonisation de ces différents habitats.

Les juvéniles recherchent particulièrement 3 habitats : les galets, la végétation aquatique et les berges, surtout celles tapissées de radicelles. A eux trois, ces habitats regroupent environ $80 \%$ de la population des juvéniles. La densité la plus élevée de juvéniles échantillonnés s'observe dans les galets, avec 6,8 individus $/ \mathrm{m}^{2}$.

Certains habitats sont peu colonisés voire même délaissés par les juvéniles, comme le sable et les graviers $\left(0,1\right.$ et 0 individu $\left./ \mathrm{m}^{2}\right)$, les souches $\left(0,3\right.$ individu $\left./ \mathrm{m}^{2}\right)$ et les débris végétaux $\left(1,4\right.$ individus $\left./ \mathrm{m}^{2}\right)$.

Les blocs présentent un cas particulier ; ils abritent non seulement $12,5 \%$ des juvéniles avec 3 individus $/ \mathrm{m}^{2}$, mais ils servent également de refuge et de zone de recherche de nourriture aux autres stades d'écrevisses, y compris les adultes (Figure 6).

\section{Répartition des juvéniles dans les habitats en fonction du cours d'eau}

La stratégie de colonisation des habitats par les juvéniles varie d'un ruisseau à l'autre (Tableaux III et IV). La comparaison par un test de Kruskal-Wallis de la fréquence des juvéniles dans les 8 habitats met en évidence des différences significatives suivant les trois cours d'eau. Ainsi, pour une valeur limite de 5,72 au seuil de signification 0,05 , nous avons 5,93 entre le Lachaud et le Vent Haut, 6,31 entre le Vent Haut et le Pont Aubert et 7,00 entre le Pont Aubert et le Lachaud.

Globalement, les juvéniles se regroupent essentiellement dans les galets (38,9\%), les berges $(22,5 \%)$, la végétation aquatique $(19,6 \%)$ et les blocs (16\%), mais de grandes variations s'observent entre les différents ruisseaux. Ainsi, les juvéniles du Vent Haut se regroupent essentiellement dans les galets (64\%), avec une densité élevée $\left(26,1\right.$ individus $\left./ \mathrm{m}^{2}\right)$, alors que dans le Lachaud ils ne sont plus que $11,9 \%$ dans cet habitat avec 3,7 individus $/ \mathrm{m}^{2}$.

La végétation aquatique, qui représente $18 \mathrm{~m}^{2}$ sur les stations du Pont Aubert (Tableau II), soit près de $8 \%$ des habitats colonisables par les écrevisses sur ce cours d'eau, abrite $13,9 \%$ de la population de juvéniles. Sur le Lachaud la végétation qui a une surface pourtant deux fois moindre abrite $44,9 \%$ de la population de juvéniles.

Les juvéniles peuvent compenser la régression ou même la disparition de certains habitats, en augmentant leur présence donc leur densité dans d'autres habitats disponibles, qui ne correspondent pas forcément à leurs préférences. Ainsi, nous voyons que :

- La réduction de la surface des galets est suivie d'une augmentation de la colonisation des amas de blocs.

- La réduction de la surface des blocs oblige les juvéniles à coloniser davantage les berges et la végétation aquatique. 
- Certains habitats normalement peu fréquentés par les juvéniles dans les ruisseaux corréziens comme les débris végétaux peuvent leur servir de refuge, si d'autres habitats font défaut. C'est le cas du Vent Haut, où l'absence de la végétation aquatique peut être compensée par la colonisation des zones de souches et de débris végétaux.

\section{Tableau III}

Densité des écrevisses juvéniles (individus $/ \mathrm{m}^{2}$ ) dans les habitats des trois ruisseaux étudiés.

\section{Table III}

Juvenile crayfish density (individuals $/ \mathrm{m}^{2}$ ) in the habitats of the three studied brooks.

\begin{tabular}{|c|c|c|c|c|c|c|c|c|}
\cline { 2 - 9 } \multicolumn{1}{c|}{} & Sable & Graviers & Galets & Blocs & Berges & Végétation & Souches & Débris \\
\hline Pont Aubert & 0,0 & 0,0 & 6,4 & 3,6 & 4,5 & 0,6 & 0,0 & 0,0 \\
\hline Lachaud & 0,0 & 0,0 & 3,7 & 1,6 & 9,3 & 1,0 & 0,0 & 2,0 \\
\hline Vent Haut & 1,1 & 0,0 & 26,1 & 7,8 & 0,0 & 0,0 & 0,6 & 5,0 \\
\hline Moyenne & $\mathbf{0 , 4}$ & $\mathbf{0 , 0}$ & $\mathbf{1 2 , 1}$ & $\mathbf{4 , 3}$ & $\mathbf{4 , 6}$ & $\mathbf{0 , 5}$ & $\mathbf{0 , 2}$ & $\mathbf{2 , 3}$ \\
\hline
\end{tabular}

\section{Tableau IV}

Répartition des écrevisses juvéniles, en pourcentage dans les habitats de chaque ruisseau.

\section{Table IV}

Percentage distribution of juvenile crayfish in the habitats of each brook.

\begin{tabular}{|c|c|c|c|c|c|c|c|c|}
\cline { 2 - 9 } \multicolumn{1}{c|}{} & Sable & Graviers & Galets & Blocs & Berges & Végétation & Souches & Débris \\
\hline Pont Aubert & 0,0 & 0,0 & 40,7 & 25,9 & 19,5 & 13,9 & 0,0 & 0,0 \\
\hline Lachaud & 0,0 & 0,0 & 11,9 & 8,1 & 34,0 & 44,9 & 0,0 & 1,1 \\
\hline Vent Haut & 4,0 & 0,0 & 64,0 & 14,0 & 14,0 & 0,0 & 2,0 & 2,0 \\
\hline Moyenne & $\mathbf{1 , 3}$ & $\mathbf{0 , 0}$ & $\mathbf{3 8 , 9}$ & $\mathbf{1 6 , 0}$ & $\mathbf{2 2 , 5}$ & $\mathbf{1 9 , 6}$ & $\mathbf{0 , 7}$ & $\mathbf{1 , 0}$ \\
\hline
\end{tabular}

\section{Colonisation des habitats par les écrevisses juvéniles et le benthos}

Lors de l'échantillonnage des écrevisses avec le filet benthique, nous avons aussi récolté des invertébrés benthiques dans les différents habitats sur les trois cours d'eau. Leurs peuplements sont principalement composés par :

- Des éphéméroptères (en majorité Baetidae, Heptagenidae, Ephemerellidae).

- Des plécoptères (surtout Leuctridae, Nemouridae).

- Des crustacés, largement dominés par des Gammaridae.

Les densités les plus élevées en juvéniles d'écrevisses (Figure 8) se retrouvent dans les galets et les habitats des berges, qui abritent aussi des densités élevées en macro invertébrés benthiques. Par contre, d'autres habitats riches en macro invertébrés comme la végétation aquatique, les graviers et les souches ne constituent pas des abris particulièrement recherchés par les écrevisses juvéniles. 

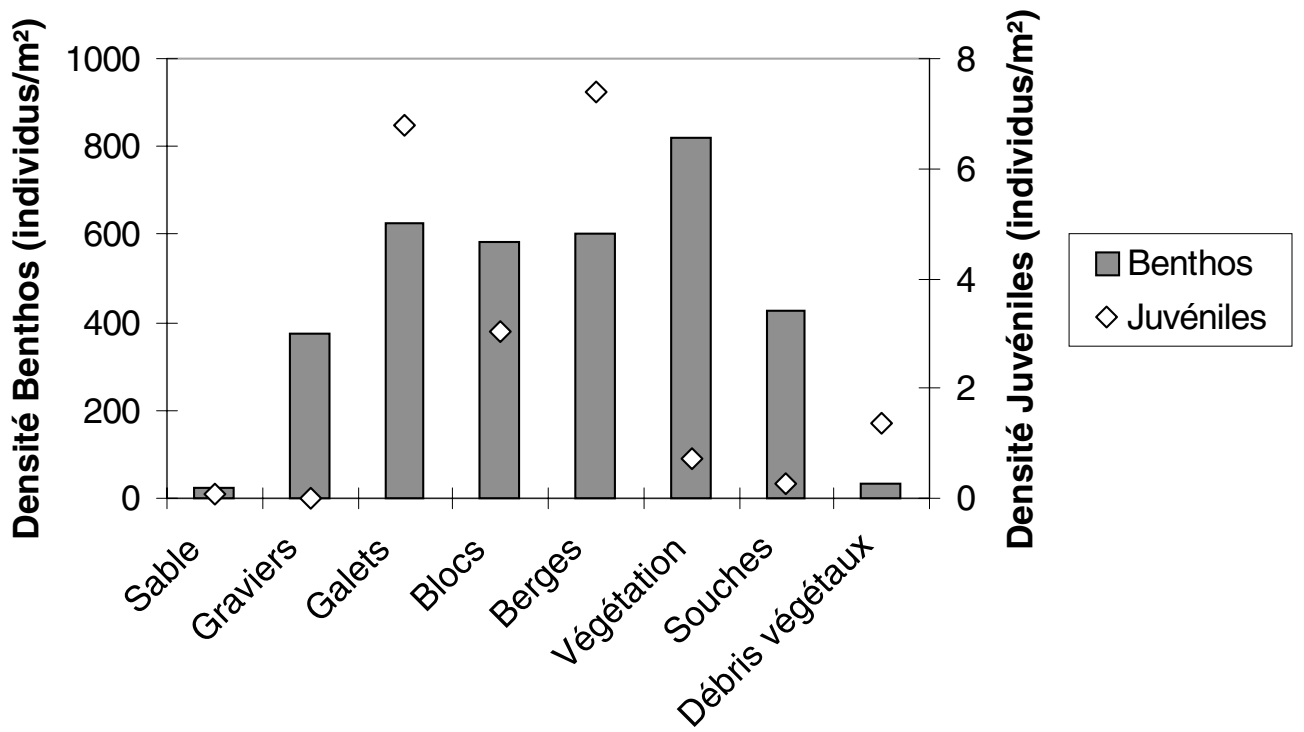

\section{Figure 8}

Densité moyenne des invertébrés benthiques et des écrevisses juvéniles (individus $/ \mathrm{m}^{2}$ ) dans les différents habitats.

\section{Figure 8}

Mean density of benthic macro-invertebrates and juvenile crayfish (individuals $/ \mathrm{m}^{2}$ ) in the different habitats.

\section{DISCUSSION}

L'utilisation de trois méthodes d'échantillonnage (le filet benthique, la pêche électrique de jour et la pêche à la main de nuit) a permis d'analyser la répartition des écrevisses à pattes blanches, en fonction de leur stade de développement, dans les habitats de 3 cours d'eau de Corrèze. Il a été également possible de suivre la fréquentation par les adultes des habitats en fonction de leur comportement: la recherche d'un refuge en cours de journée et les déplacements nocturnes pour l'alimentation.

\section{Habitats et comportement des écrevisses adultes}

Globalement, les écrevisses adultes peuvent utiliser tous les habitats étudiés dans les ruisseaux corréziens. Cependant, certains d'entre eux semblent correspondre à une activité bien particulière des écrevisses. Ainsi, $25 \%$ des adultes capturés dans les graviers sont en déplacement, alors qu'ils ne sont plus que $3 \%$ à y rechercher un abri en cours de journée.

Les habitats fortement colonisés par les écrevisses adultes durant la journée sont les berges constituées de racines ou avec des cavités, les blocs, les galets, la végétation aquatique et les souches. L'habitat de type bloc semble être particulièrement intéressant pour les adultes ( $28 \%$ des écrevisses échantillonnées en déplacement nocturne et $31 \%$ de jour), car il constitue à la fois un abri et un milieu favorable pour la recherche de la nourriture. L'affinité des écrevisses à pattes blanches pour les zones de blocs et de galets a déjà été remarquée (FOSTER, 1995 ; BROWN et BOWLER, 1997 ; NAURA et ROBINSON, 1998) et pourrait être mise en relation avec la stabilité de cet habitat, la 
fourniture de nombreuses caches, ainsi que par la forte production de périphyton et de macro invertébrés qui y est associée.

Les densités d'écrevisses adultes capturées dans les habitats utilisés comme abris sont en moyenne proches de 1 individu $/ \mathrm{m}^{2}$. Cette valeur est inférieure aux estimations de densités calculées sur d'autres cours d'eau et basées sur des pêches successives avec marquage et recapture des écrevisses (ROQUEPLO, 1983). Les densités de capture les plus élevées dans les ruisseaux corréziens s'observent dans les blocs $\left(1,3 \mathrm{individu} / \mathrm{m}^{2}\right)$, les souches $\left(1 \mathrm{individu} / \mathrm{m}^{2}\right)$ et les berges $\left(0,8 \mathrm{individu} / \mathrm{m}^{2}\right)$. L'habitat de type souche, qui abrite des densités d'adultes satisfaisantes, n'est fréquenté que par $4 \%$ de la population. Cette faible valeur est à mettre en relation avec la faible représentation de cet habitat, qui ne constitue que $1,2 \%$ de la surface totale des différents habitats recensés.

\section{Habitat préférentiel des juvéniles}

Si les écrevisses, toutes tailles confondues, fréquentent bien les 8 habitats que nous avons identifiés, certains d'entre eux apparaissent particulièrement attractifs pour les juvéniles.

Ceux-ci se regroupent surtout dans les galets, les blocs, les végétaux aquatiques et les berges. Parmi les différents types de berges échantillonnés, les juvéniles colonisent en majorité les chevelus de fines racines. Par contre, les juvéniles ne se rencontrent que rarement dans le sable, les graviers, les souches et les débris végétaux.

Les souches ne servent d'abris qu'à de rares juvéniles, probablement parce qu'elles sont fréquentées par de fortes densités d'écrevisses de moyenne et de grande taille (ROQUEPLO, 1983), capables de prédation sur les juvéniles.

Dans les ruisseaux étudiés en Corrèze, certains habitats sont faiblement représentés par rapport à ce qui a pu être trouvé dans d'autres cours d'eau. Par exemple, les débris végétaux ne sont représentés que par de très faibles surfaces $(2,3 \%$ des habitats), alors que dans d'autres sites des amas de feuilles constituent des surfaces importantes et abritent une large proportion de la population de juvéniles (ARRIGNON et ROCHE, 1981).

Des essais sur de jeunes Pacifastacus leniusculus (MASON, 1978) ont montré que leur taux de survie ainsi que leur croissance pondérale augmentent significativement si la rugosité du substrat augmente, par exemple en passant du sable aux galets. Ceci est à mettre en relation avec la plus grande disponibilité des interstices entre les galets. L'augmentation de la taille des éléments constituant le substrat limite considérablement le taux de prédation des poissons carnassiers sur les écrevisses (STEIN et MAGNUSSON, 1976). Cependant, si la granulométrie est faible, les poissons carnassiers consomment en priorité les plus petites écrevisses (STEIN, 1977). Par contre, en présence de galets ou de blocs, le prédateur va chercher en priorité des écrevisses de plus grande taille, dont la capture semble plus rapide ; les jeunes individus semblent donc être bien protégés par les nombreux interstices crées par ce type d'habitat, et la présence de zones de galets et de blocs dans les ruisseaux de Corrèze est donc un facteur favorable pour la pérennité des populations de $A$. pallipes.

Les berges, de par leur architecture, sont des structures de rétention de la nourriture allochtone, protègent efficacement les écrevisses lors des crues (SMITH et al., 1995) et pourraient servir de zones de refuge, au même titre que les souches. Compte tenu de la diversité des types de berge que nous avons rencontrés dans les ruisseaux de Corrèze, il est possible que cet habitat serve d'abri à un nombre important d'écrevisses de tous âges. 
Des études antérieures ont d'ailleurs montré que les berges jouent un rôle important pour la stabilité des populations d'écrevisses en cours d'eau (FOSTER, 1995 ; SMITH et al., 1995 ; ROQUEPLO, 1997) et attestent du rôle primordial de cet habitat.

Les densités les plus fortes d'invertébrés benthiques s'observent dans les galets, les blocs, les végétaux aquatiques et les berges, au contraire du sable et des débris végétaux, ce qui est conforme à ce qui a été trouvé sur la répartition à grande échelle des macro invertébrés en cours d'eau (MINSHALL et ROBINSON, 1998). Dans la revue bibliographique qu'il a réalisée, GODDARD (1988) montre que les écrevisses juvéniles des genres Austropotamobius et Astacus ont un régime alimentaire essentiellement carnivore constitué en majorité d'invertébrés aquatiques. Les densités élevées d'écrevisses juvéniles dans certains habitats, comme les galets, peuvent donc s'expliquer non seulement par la structure même du milieu (interstices, stabilité, sous écoulement), mais également par la proximité d'une source de nourriture abondante. Les faibles densités observées dans les macrophytes, pourtant colonisés par de fortes densités de macro invertébrés, peuvent sembler surprenantes. La forte concentration de Gammares dans cet habitat est peut être la source des faibles densités observées, du fait d'une possible moins bonne qualité nutritive. II faut cependant rappeler que, même si les densités de juvéniles observées dans cet habitat restent faibles, c'est dans celui-ci que le plus grand nombre d'individus a été capturé. II doit donc être considéré comme indispensable dans la définition de l'habitat préférentiel des juvéniles, au même titre que les galets, les blocs et les berges constituées de fines racines.

\section{CONCLUSION - PERSPECTIVES}

Les zones colonisées par les écrevisses à pattes blanches dans les ruisseaux de Corrèze peuvent être considérées comme une mosaïque d'habitats. Parmi ceux-ci, certains types de berges, les souches, les blocs et les bancs de galets semblent être indispensables à l'installation et à la pérennité d'une population abondante d'écrevisses à pattes blanches. Des études sur la colonisation des habitats par les écrevisses (SMITH et al., 1995) ont montré que la juxtaposition de plusieurs paramètres (l'ombrage, la proportion de berges verticales, de racines dans les berges et la couverture herbeuse des berges) peut expliquer entre 70 et $85 \%$ de la distribution des écrevisses dans les cours d'eau étudiés. Une fois que les habitats colonisés par les écrevisses sont bien identifiés, il est par la suite important d'avoir une vision plus globale et de suivre la colonisation d'un cours d'eau à l'échelle de structures morphologiques ayant des caractéristiques de vitesse et de profondeur homogènes (NEVEU, 2000).

II est difficile de mettre en avant une quelconque hiérarchie des différents habitats favorables au maintien d'une population d'écrevisses à pattes blanches. La disparition de certains d'entre eux, ou leur faible représentation dans un secteur de cours d'eau pouvant amener les écrevisses, et en particulier les juvéniles, à occuper des habitats qu'ils ne fréquentent pas habituellement. C'est le cas de la colonisation des souches et des débris végétaux par les juvéniles, du fait de la réduction des bancs de galets, du faible développement des végétaux aquatiques ou de la petite quantité d'abris dans les berges.

Les populations de $A$. pallipes, et en particulier les juvéniles, peuvent donc s'adapter, dans une certaine mesure, à des modifications dans la répartition spatiale des habitats qu'elles colonisent. C'est un facteur encourageant, qui pourrait expliquer en partie le maintien de populations d'écrevisses à pattes blanches dans de nombreux cours d'eau. Mais il reste à savoir dans quelle mesure la modification, la régression ou la disparition de certains habitats peut modifier le taux de recrutement et induire progressivement un affaiblissement de leurs populations, voire même leur extinction. 


\section{BIBLIOGRAPHIE}

ARRIGNON J., 1990. L'enquête nationale sur les écrevisses; une réglementation inadaptée. Eaux Libres, 2, 3-8.

ARRIGNON J., ROCHE B., 1981. Population of the crayfish Austropotamobius pallipes pallipes in a brook of Corsica, France. Freshwater Crayfish, V, 229-238.

BREWIS J.M., 1978. Dynamics of a population of the freshwater crayfish Austropotamobius pallipes (Lereboullet). Freshwater Crayfish, IV, 153-158.

BROWN D.J., BOWLER K., 1976. A population study of the british freshwater crayfish Austropotamobius pallipes (Lereboullet). Freshwater Crayfish, III, 33-50.

DAGUERRE DE HUREAUX N., ROQUEPLO C., 1981. Définition du biotope préférentiel de l'écrevisse à pattes blanches, Austropotamobius pallipes (Ler.) dans un ruisseau landais. Bull. Fr. Piscic., 281, 211-222.

DAVENNE P., FIRMIGNAC F., 1997. L'habitat potentiel de l'écrevisse à pieds blancs et la ripisylve dans la Vallée Française (Lozère). Inventaires et propositions de gestion. Rapport de maîtrise GRED, Université Clermont-Ferrand II, 89 p.

FENOUIL E., 1987. Biologie et développement de l'écrevisse Austropotamobius pallipes pallipes (Lereboullet, 1858) en région provençale. Thèse Doc. ès Sciences, AixMarseille, $291 \mathrm{p}$.

FENOUIL E., CHAIX J.C., 1985. Cycle biologique et comportement d'une population d'Austropotamobius pallipes (Lereboullet, 1858). Ecologia Mediterranea, XI (2/3), 3-23.

FOSTER J., 1995. Factors influencing the distribution and abundance of the crayfish Austropotamobius pallipes in Wales and the Marches, UK. Freshwater Crayfish, VIII, 79-98.

FRONTIER S., 1983. Stratégies d'échantillonnage en écologie. Masson, Collection d'écologie, $495 \mathrm{p}$.

GODDARD, 1988. Food and feeding. In : HOLDICH D.M. and LOWERY R.S. (Eds.), Freshwater Crayfish: Biology, Management and Exploitation. Croom Helm, London, $498 \mathrm{p}$.

HOGGER J.B., 1988. Ecology, population biology and behaviour. In : HOLDICH D.M. and LOWERY R.S. (Eds.), Freshwater Crayfish: Biology, Management and Exploitation. Croom Helm, London, 498 p.

LACHAT G., LAURENT P.J., 1987. The habitats of Astacus astacus (L.) and Austropotamobius pallipes Lere. in the Morvan. Freshwater Crayfish, VII, 61-68.

MACAN T., 1958. Methods of sampling the bottom fauna in stony streams. Mitt. Internat. Verein. Limnol., 8, 1-21.

MASON J.C., 1978. Effects of temperature, photoperiod, substrate, and shelter on survival, growth and biomass. Freshwater Crayfish, IV, 73-82.

MINSHALL G.W., ROBINSON C.T., 1998. Macroinvertebrate community structure in relation to measures of lotic habitat heterogeneity. Arch. Hydrobiol., 141 (2), 129-151.

NAURA M., ROBINSON M., 1998. Principles of using river habitat survey to predict the distribution of aquatic species: an example applied to the native white clawed crayfish Austropotamobius pallipes. Aquatic Conserv. Mar. Freshw. Ecosyst., 8, 515-527.

NEVEU A., 1996. Caractéristiques démographiques de stocks résiduels de l'écrevisse à pattes blanches Austropotamobius pallipes (Astacidae), en Normandie. Cybium, 20 (3) sup., 75-93.

NEVEU A., 2000. Etude des populations d'Austropotamobius pallipes (Crustacea, Astacidae) dans un ruisseau forestier de Normandie. II. Répartition en fonction de la structure des habitats ; stabilité et variabilité au cours de cinq années. Bull. Fr. Pêche Piscic., 356, 99-122.

ROQUEPLO C., 1983. Etudes de populations naturelles d'écrevisses (Austropotamobius pallipes Ler.) dans le Sud-Ouest de la France. Cemagref. Etude 14, $180 \mathrm{p}$. 
ROQUEPLO C., 1992. Etudes des populations d'écrevisses en milieu naturel : les principales méthodes de capture. Informations techniques du Cemagref, 88 (5), 1-7.

ROQUEPLO C., 1997. Etude de populations d'écrevisses à pattes blanches (Austropotamobius pallipes Ler.) en cours d'eau, soumises à une pêche de loisir. L'Astaciculteur de France, 51, 59-68.

ROQUEPLO C., 1999. L'écrevisse à pattes blanches dans les cours d'eau de Corrèze. Rapport Cemagref, $70 \mathrm{p}$.

SMITH G.R.T., LEARNER M.A., SLATER F.M., FOSTER J., 1995. Habitat features important for the conservation of the native crayfish Austropotamobius pallipes in Britain. Biol. Conserv., 75, 239-246.

STEIN R.A., 1977. Selective predation, optimal foraging, and the predator-prey interactions between fish and crayfish. Ecology, 58, 1237-1253.

STEIN R.A., MAGNUSON J.J., 1976. Behavioral response of crayfish to a fish predator. Ecology, $57 n^{\circ} 4,751-761$.

WESTMAN K., SUMARI O., PURSIANEN M., 1978. Electric fishing in sampling crayfish. Freshwater Crayfish, IV, 251-256. 